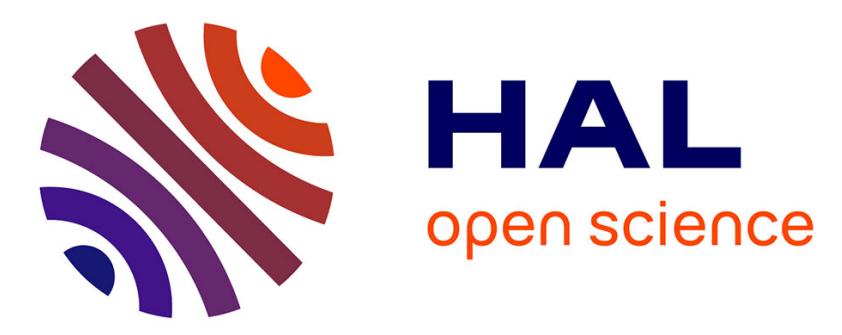

\title{
High resolution LDA measurements in transitional oblique shock wave boundary layer interaction
}

\author{
Moussa Diop, Sébastien S. Piponniau, Pierre Dupont
}

\section{To cite this version:}

Moussa Diop, Sébastien S. Piponniau, Pierre Dupont. High resolution LDA measurements in transitional oblique shock wave boundary layer interaction. Experiments in Fluids, 2019, 60 (4), 10.1007/s00348-019-2701-x . hal-02415026

\section{HAL Id: hal-02415026 \\ https://hal.science/hal-02415026}

Submitted on 2 Jul 2020

HAL is a multi-disciplinary open access archive for the deposit and dissemination of scientific research documents, whether they are published or not. The documents may come from teaching and research institutions in France or abroad, or from public or private research centers.
L'archive ouverte pluridisciplinaire HAL, est destinée au dépôt et à la diffusion de documents scientifiques de niveau recherche, publiés ou non, émanant des établissements d'enseignement et de recherche français ou étrangers, des laboratoires publics ou privés. 


\title{
High resolution LDA measurements in transitional oblique shock wave boundary layer interaction
}

\author{
Moussa Diop · Sébastien Piponniau - Pierre Dupont
}

Received: date / Accepted: date

\begin{abstract}
Spatial development of a transitional Oblique Shock Wave Interaction at Mach 1.68 is presented. This type of flow is characterised by very small length scales (boundary layer thickness is smaller than $1 \mathrm{~mm}$ ), high velocities, reverse flows and a wide range of velocity fluctuations along the transition process. Unsteady velocity fields have been obtained using a high spatial resolution Laser Doppler Anemometry system, allowing quantitative measurements of the velocity fluctuations down to $y / \delta=0.1$. A model to take into account the finite size of the probe volume on the mean and RMS velocity measurements is used and applied to the present measurements. Finally, the amplification of the velocity fluctuations along the transitional separated shear layer is described.
\end{abstract}

Keywords LDA - Supersonic flow - Transitional · Shock wave boundary layer interaction

\section{Introduction}

A challenge for the aeronautical industries is currently the reduction of greenhouse gases and, consequently, the aerodynamic design of the different parts of a plane, from wings to internal engine parts, has to be more and more efficient in order to reduce fuel consumption. A

Moussa Diop

Aix Marseille Univ, CNRS, IUSTI, Marseille, France

Sébastien Piponniau

Aix Marseille Univ, CNRS, IUSTI, Marseille, France

E-mail: sebastien.piponniau@univ-amu.fr

Pierre Dupont

Aix Marseille Univ, CNRS, IUSTI, Marseille, France promising way to achieve this is to reduce the viscous drag by the use of laminar wings. This is why transonic aviation is currently exploring the possibility of airplanes flying at higher altitude, where laminar conditions and skin friction reduction are more easily obtained.

Transonic flows are characterised by the formation of local supersonic zones on the external parts of the wings or in the first stages of a jet engine compressor for example, and are associated with the formation of shock waves. The interaction between the laminar boundary layer and the shock wave is thus of primary importance, since these kinds of interactions are known to develop large separated regions and promote the transition to turbulence of the incoming boundary layer [11]; [28].

Shock Wave Boundary Layer Interactions (SWBLI) have been extensively studied these past decades, with a focus on configurations where the upstream boundary layer is turbulent. These flows are critical for numerous high speed applications, such as supersonic intakes or over-expanded nozzle, since the interaction can generate thermal and aerodynamic loads that can be harmful for the system ([10]). Pioneers' investigations can be found in the earlier $1950 \mathrm{~s}([1,18])$, where results were focused on the spatial description of the interaction and on the pressure description. More recent research has mainly focused on the source of the low frequency unsteadiness observed: when the shock strength is strong enough to make the boundary layer separate, these interactions are characterised by a strong low-frequency unsteadiness of the separation bubble and the separation shock, while the associated characteristic frequency is two orders of magnitude lower than the characteristic 
frequencies of the incoming boundary layer.

When addressing the problem of the shock wave boundary layer interaction involving a laminar or transitional boundary layer in a supersonic flow, the literature is somewhat limited in comparison with the turbulent case or rather old with regard to new measurements techniques. The Work of Chapmann et al. [3] has shown that these interactions can be divided in two major cases: when the transition occurs along the interaction, or downstream of the interaction. In the latter case, Chapmann has shown that the interaction is statistically steady, while unsteadiness appears when transition to turbulence occurs along the interaction. Delery [17] shows that the transition position of the boundary layer tends to move upstream by increasing the Reynolds number: the transition location moves from the downstream flow to a position close to the incident shock impingement. Then, a further increase in the Reynolds Number does not affect the location of transition until a critical Reynolds number, at which point the transition location suddenly occurs upstream of the separation. Liepman [19] has shown that the aspect ratio of the separation (length over height) is quite large : about 50 for a transitional interaction, compared to an aspect ratio of about 10 for the turbulent case.

New experiments were conducted to describe spatially laminar and/or transitional SWBLI, using Particle Image Velocimetry (PIV) measurements ([12,11, 10]) as well as numerical simulations $[27,5,16]$, using LES or DNS. These studies have shown that the laminar SWBLI is subjected to turbulence transition along the separated boundary. Depending on the shock intensity this transition can appear either at the impingement position of the incident shock wave, for high shock intensities, or further downstream, for low shock intensities. The first case is characterised by an abrupt reattachment of the separated boundary layer, whereas in the second case the reattachment is much smoother and the interaction length longer.

The experimental investigation of such flows is quite challenging : laminar boundary layers are very thin, less than $1 \mathrm{~mm}$ in the present experimental configuration, associated with high velocity gradient. By nature, laminar boundary layers do not promote mixing: thus, velocity measurements based on flow seeding are particularly delicate, due to the non-uniformity of the seeding in the flow ([12]). For example, as in the recent work by Giepman et al. [12], classical PIV algorithms failed to resolve instantaneous velocity due to the poor seeding density. The mean quantities could be obtained by ensemble correlation approach [21], but turbulent fields are not accessible. Even with high res-

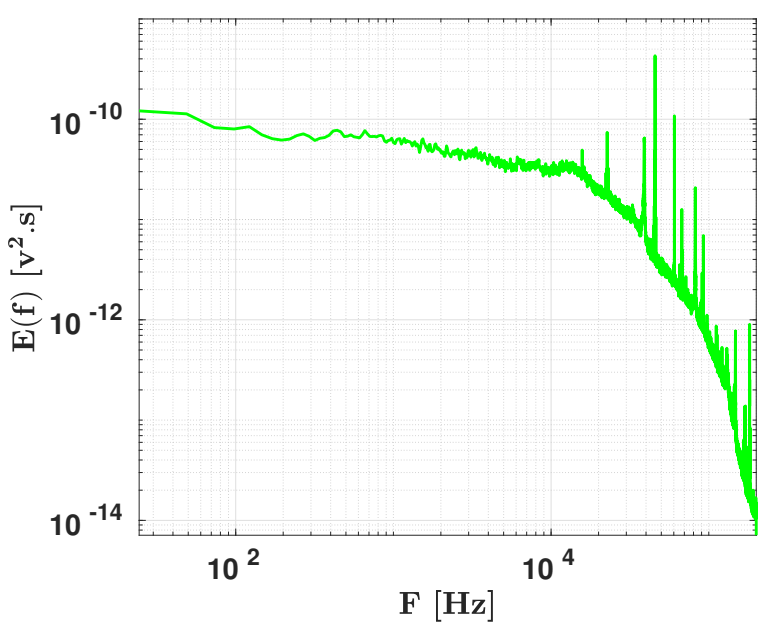

Fig. 1 Velocity spectrum in the upstream flow, $P_{0}=0.4 \mathrm{~atm}$

olution macro-PIV measurements, the boundary layer thickness $\delta(\approx 0.2 \mathrm{~mm})$ is described by 26 pixels. With regard to the interrogation windows used, this leads to a spatial resolution of $h / \delta=0.31$, where $h=62 \mu \mathrm{m}$ is the height of the PIV interrogation windows (8 pixels, without Gaussian weighting).

This paper presents the experimental set-up for an oblique shock wave / Laminar Boundary layer interaction at a Mach number of 1.68 , for a limited range of unit Reynolds numbers (5e6 to 11e6). An improved LDA set-up will be shown in order to provide high spatial resolution and quantitative longitudinal mean velocity measurements of the incoming boundary layer or inside the interaction, with spatial resolution of $h / \delta=$ 0.068. The measurement accuracy will be addressed, and a simple model for evaluating bias due to integration effects of the measurement volume in the velocity measurements, based on those proposed by $[13,22,15$, 9 ], will be presented and tested on the incoming laminar boundary layer measurements. Finally, a detailed description of the interaction by LDA measurements will be presented and the longitudinal amplification of velocity fluctuations from the upstream laminar boundary layer to the turbulent reattachment will be characterised.

\section{Experimental setup}

\subsection{Facility overview}

Experiments are performed in the S7 test section of the supersonic wind tunnel at the Institut Universitaire 


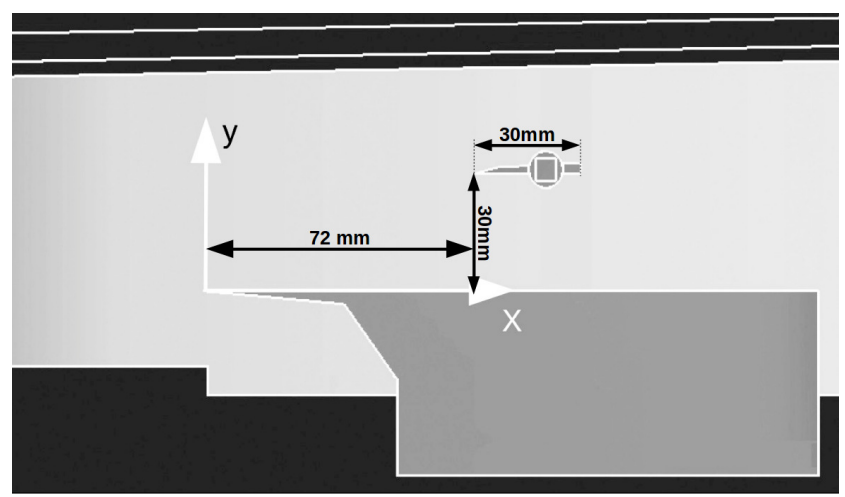

Fig. 2 Test section with model

des Systèmes Thermiques Industriels (IUSTI) in Marseille. It is a closed-loop facility, operating continuously in hypo-turbulent conditions. It may be operated up to four hours without pressure drift, for stagnation pressures ranging from 0.15 to $0.9 \mathrm{~atm}$. The nominal Mach number is 1.68 . The pressure fluctuations in the potential flow are as low as $0.4 \%$ at a stagnation pressure of $0.4 \mathrm{~atm}$. The spectrum of velocity fluctuations in the external flow, obtained by Hot Wire Anemometry at the inlet of the test section, is presented in Figure 1. The stagnation temperature depends on the ambient temperature. It is maintained constant with a drift of less than $1 K /$ hour thanks to a cooling water system. The working section is $150 \mathrm{~mm}$ wide (span-wise) and $80 \mathrm{~mm}$ high.

This study investigates a laminar supersonic boundary layer developing over a flat plate on which an oblique shock wave impinges. The model (see Figure 2) is composed of a flat plate of $175 \mathrm{~mm}$. Its leading edge is placed at $110 \mathrm{~mm}$ from the exit of the wind tunnel nozzle and raised up at $20 \mathrm{~mm}$ distance from the bottom wall in order to avoid the floor boundary layer of the wind tunnel interacting with the studied laminar boundary layer. A sharp-edged shock generator of $30 \mathrm{~mm}$ length is installed at $72 \mathrm{~mm}$ downstream of the leading edge of the flat plate, and at $30 \mathrm{~mm}$ above the plate. The shock generator and the flat plate span the width of the wind tunnel test section. The origin of the abscissa $x$ is taken at the leading edge of the flat plate.

For this Mach number, special attention must be paid to reducing the risks of blockage effects: these were minimised during the aerodynamic design of the test section using 2D RANS simulations. Consequently, the leading edge of the flat plate is less than 5 degrees and a cavity is made underneath of the flat plate to avoid chocking this secondary channel. A progressive recompression is generated downstream of the plate.

The plexiglass wind tunnel windows are $60 \mathrm{~mm}$ thick with an optical quality that is not good enough to allow

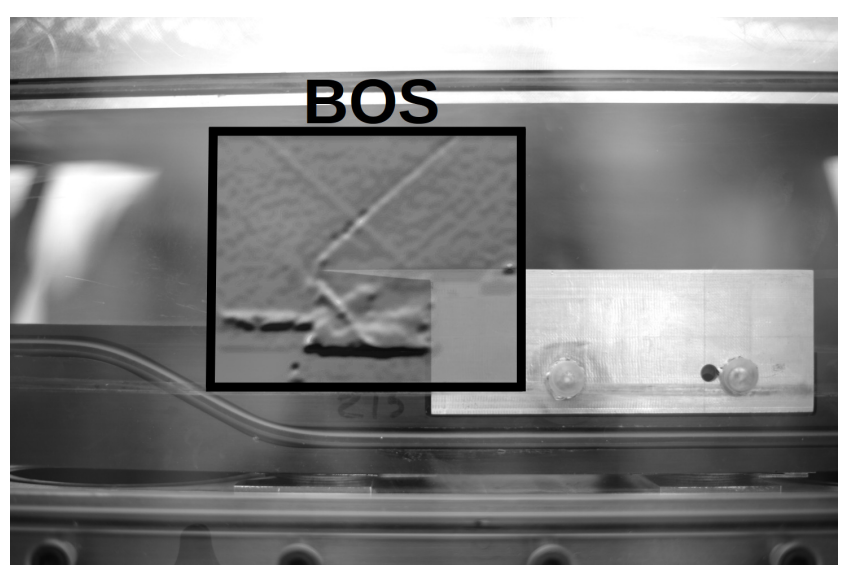

Fig. 3 BOS visualisation of the flow around the flat plate (without the shock generator) superimposed to a picture of the test section

classical Schlieren visualisations. Therefore, flow visualisations have been achieved using a Background Oriented Schlieren (BOS) system [25]. The BOS Method is a very simple technique for visualising density gradients, and, contrary to schlieren visualisations, does not necessarily need high quality optical windows. It is based on the deviation of light rays due to refractive index changes, but with no needs of dedicated lenses or mirror. A background image of a random dot pattern is used and a reference image is taken through the test section with no flow. Then, a second image of the background is taken when the flow has been established. The resulting images can then be evaluated by classical image correlation methods. Here, we use the PIV correlation algorithm from Dynamic Studio (Dantec Dynamics). The displacement map is directly linked to the density gradient in the flow, e.g. the shock and expansion waves in our particular configuration. Figure 3 shows the flat plate and the BOS visualisation is superimposed along the leading edge. A slight oblique shock wave is observed at the leading edge of the plate. Under the plate, the shock wave and the expansion wave emanating respectively from the leading edge and from the cavity corner are clearly visible. Without the shock generator installed, there is almost certainly no blockage effects in the test section.

The shock generator is directly housed in the plexiglass windows: this avoids the presence of masts to fix the generator which would cause additional blockage. Unfortunately, this led to some optical faults in the plexiglass windows. Moreover, when the flow starts, the low pressure inside the test section generates some mechanical stresses around the fixations which prohibited satisfactory BOS visualisations. Therefore, the interaction's organisation in space was derived from pitot probe measurements (see section 2.2). 


\subsection{Oblique shock wave interaction}

The expected SWBLI organisation is illustrated in Figure 4 . The stagnation pressure $P_{0}$ and the flow deviation $\theta$ were respectively fixed to $0.4 \mathrm{~atm}$ and 5 degrees. As mentioned in the previous section, the flow organisation was obtained by Pitot pressure measurements. A Pitot probe was placed outside of the shear layer ( $5 \mathrm{~mm}$ over the wall). The location and pressure step across the upstream compression waves and of the incident shock wave have been derived from longitudinal exploration along the interaction (see Figure 4). It was then possible to determine the associated flow deviations and finally to extrapolate down to the wall the measurements achieved over the plate.

The impingement location of the incident shock wave $X_{i m p}$ is at $107.3 \mathrm{~mm}$ from the leading edge. At this location, the undisturbed boundary layer thickness is $\delta_{i m p}=0.9 \mathrm{~mm}$ and the displacement thickness is $\delta_{i m p}^{*}=$ $0.4 \mathrm{~mm}$. The upstream influence of the interaction $X_{0}$ has been defined as the inflection point of the pressure rise across the compression waves. At this location, the undisturbed boundary layer thickness is $\delta_{0}=0.7 \mathrm{~mm}$ and the displacement thickness is $\delta_{0}^{*}=0.3 \mathrm{~mm}$. The length of the interaction $L$, defined as the distance between $X_{0}$ and $X_{i m p}$, is of $43.7 \mathrm{~mm}$. The non-dimensional longitudinal coordinate is $X^{*}=\left(X-X_{i m p}\right) / L$. The position of the incident shock at the wall is then $X^{*}=0$ and the compression waves are centered around $X^{*}=-1$. Downstream from the compression waves, the plateau region, a distinctive feature of separated interactions, is observed up to the incident shock wave. Further downstream, the expansion waves are followed by the compression waves due to the flow turning associated with reattachment of the flow.

\subsection{Laser Doppler Anemometry}

\subsubsection{Optical set-up}

Laser Doppler Anemometry will be used to describe the interaction as well as the incoming boundary layer. As the boundary layer is expected to be very thin, a single component system has been used in order to allow very near-wall measurements. An Argon-ion laser from Spectra-Physics (6W, Stabilite 2017) has been used. Only the most energetic wave length $\left(\lambda_{b}=514.5 \mathrm{~nm}\right)$ is considered for measuring the longitudinal component of the velocity. A FiberFlow system, from Dantec Dynamics, allows splitting the laser beam and the $40 \mathrm{M} \mathrm{Hz}$ frequency shift in order to resolve negative velocity. The resulting emitted beam is $2.2 \mathrm{~mm}$ in diameter. The LDA system used for these experiments is based on classical
LDA components, optimised for near wall high speed flow measurements [2]. It integrates:

- a beam spacer, to adjust the distance between the two laser beams;

- a beam expander, to increase the diameter of each beam;

- a converging lens.

The probe volume characteristics (diameter $\Phi$, length $l_{\phi}$, interfringe $\delta_{f}$ and fringes number $N_{\phi}$ ) are given by the classical relations [9]:

$\Phi=\frac{d_{f}}{\cos \left(\theta_{b} / 2\right)}, \delta_{f}=\frac{\lambda_{b}}{2 \sin \left(\theta_{b} / 2\right)}$

$N_{f}=\frac{\Phi}{\delta_{f}}$, with $d_{f}=\frac{4 f_{l} \lambda_{b}}{\pi E_{\text {exp }} d_{I}}$

where $\theta_{b}$ is the angle between the two laser beams, $f_{l}$ the focal of the converging lens, $\lambda_{b}$ the wavelength of the laser, $d_{I}$ the initial diameter of the laser beam, $d_{f}$ the laser beam diameter at the focal point and $E$ the beam-expander ratio. In our configuration, the beam diameter is $2.2 \mathrm{~mm}$, the beam distance has been set to $38 \mathrm{~mm}$, we use a beam expander with a ratio of 1.98 , and a converging lens of $500 \mathrm{~mm}$. The measurement volume is then of a diameter of $75 \mu \mathrm{m}$, a length of $1.9 \mathrm{~mm}$, and the fringe spacing is of $6.7 \mu \mathrm{m}$ : in this configuration, the maximum Doppler frequency expected for this flow is of $\approx 110 \mathrm{MHz}$. The receptive head is positioned for back-scatter, off-axis. The off-axis angle is about $25^{\circ}$, in order to receive the maximum light emanating from particles in the backward configuration and then to optimise the signal to noise ratio. Tracer particles are generated by incense smoke, whose average particle size is less than one micrometer [24]. The particle response time is estimated as $t_{p} \simeq 5 \mu \mathrm{s}$ corresponding to a cut-off frequency of about $200 \mathrm{kHz}$. In the laminar boundary layer, the expected time scales $t_{f}$ are of the order of $\mathrm{O}(10 \mathrm{kHz})$. Inside the interaction, if the separated shear layer is considered, an estimation of the most unsteady modes can be derived from the Strouhal number observed in laminar mixing layers: $S_{t}=\mathrm{O}(0.1)$ with $S_{t}=f \delta / U_{c}$. With $\delta \simeq 1 \mathrm{~mm}$, $U_{c} \simeq U_{e} / 2=225 \mathrm{~ms}^{-1}$, we have $f=O(20 \mathrm{kHz})$. Therefore, the Stokes number (Stokes $\left.=t_{p} / t_{f}\right)$ is always less than 0.1 , and the particle lag effects should not be significant[26]. Downstream from the reattachment, in the turbulent region, the thickness of the boundary layer is increased but the energetic time scale of the flow has to be derived from classical turbulent scaling (ie: $\left.t_{f} \simeq \delta / U_{e}=2 \times 10^{-3} / 450=4.4 \times 10^{-6}\right)$ and the Stokes number is about 1 . This suggests that, in this region, highest frequencies are probably attenuated due to the particle lag. Nevertheless, for the scope of this paper, 


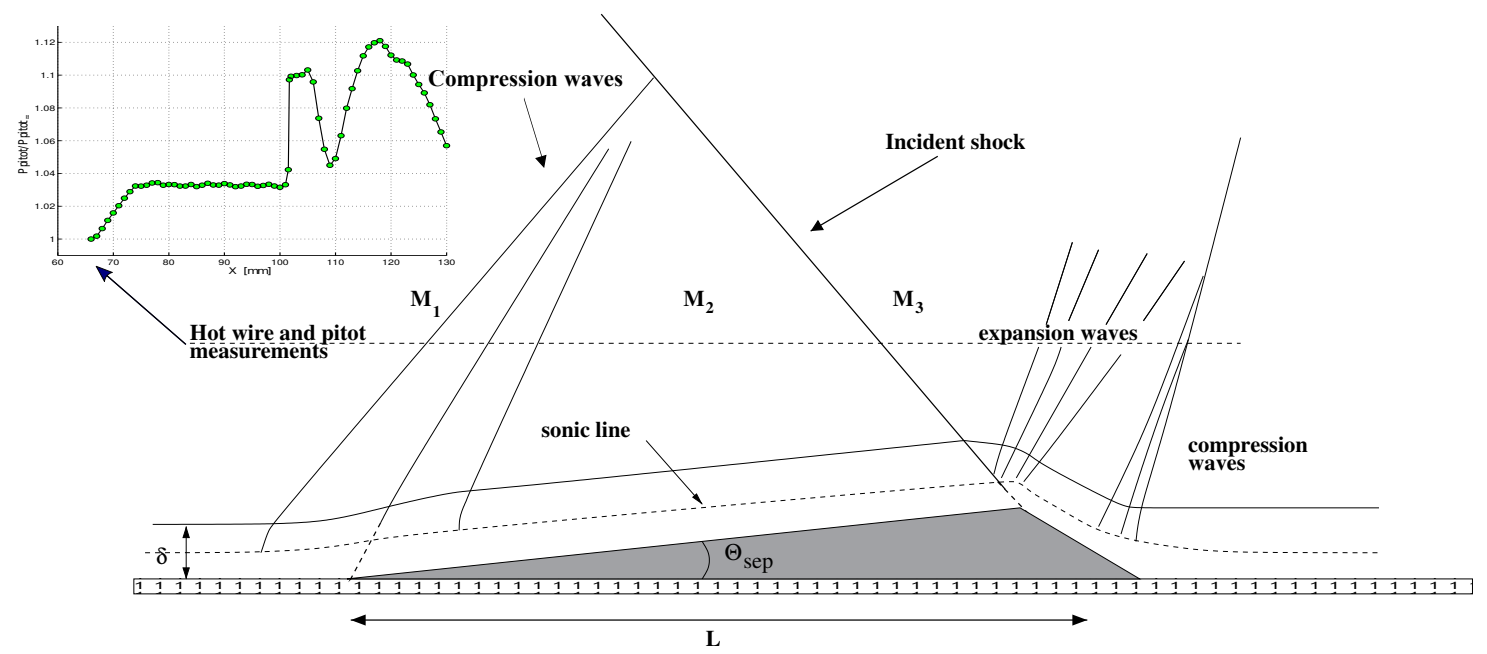

Fig. 4 Sketch of a separated laminar boundary layer interaction and longitudinal Pitot pressure through the interaction, measured at $5 \mathrm{~mm}$ from the wall. $P_{0}=0.4 a t m, \theta=5^{\circ}$. Adapted from Délery and Marvin [7]

which focuses on the transition process, the seeding is adequate. Particles are seeded through a seeding cane placed at the end of the plenum chamber, on the wind tunnel axis. This cane is adjustable vertically to seed the whole interaction zone.

Both transmitting and receiving optics are on the same displacement system. We used a Newport displacement system for longitudinal and vertical displacement with a positional accuracy, given by the manufacturer, of $1 \mu \mathrm{m}$. In this configuration, the alignment of the receptive head with the measurement volume is maintained during an acquisition. Adjustment of the alignment of receptive head is checked for each measurements points, by maximising the data rate and the burst validation. Thanks to the continuously blowing wind tunnel, 2000 samples are recorded. The number of samples is reduced in the case of very low seeding region, due to the lower rate of seeding. In LDA measurements, velocity bias can be observed when significant correlation exist between the instantaneous velocity and the seeding of the flow. Indeed, high velocity can be overestimated in comparison with low velocity events [20]. This occurs generally for high density seeding. In our case, even for the highest datarate $(\mathrm{O}(1 \mathrm{kHz}))$, the average distance is $(\mathrm{O}(40 \mathrm{~cm}))$ which is more than $400 \delta$, where $\delta$ is the boundary layer thickness in our configuration. This means that, whatever the measurement location, we are in the general configuration of very low seeding. In this case, no significant correlation is observed between the velocity and time between burst. Therefore, no correction in bias is applied.

\subsubsection{Spatial integration problems for the $L D A$ measurements}

LDA measurements in such flows are quite challenging. The LDA probe volume diameter $(\Phi=75 \mu \mathrm{m})$ has to be compared with the thickness of the boundary layer which is as small as $700 \mu \mathrm{m}$. Mean velocity measurements achieved in the laminar boundary layer for various stagnation pressures are reported in Figure 5 (see $[8])$. In the first part of the laminar boundary layer ( $\left.0<U / U_{e}<0.6\right)$ the velocity profile is assumed to be linear with a constant gradient $\alpha$. In this part of the boundary layer, the velocity gradient is then of the order of $U_{e} / \delta$. The variation of velocity $\Delta U$ across the probe volume is of the order of $U_{e} \Phi / \delta$, which shows that $\Delta U / U_{e} \simeq \Phi / \delta \simeq 0.1$. Therefore, the velocity gradient across the diameter of the probe volume cannot be neglected. In the case of a larger unit Reynolds number, these estimations are even worse, as the thickness of the boundary layer decreases as $\sqrt{R e_{u}}$. The aim of these experiments is to provide reliable measurements of the mean and fluctuations velocity along the separated shear layer.

The expected accuracy/limitations of such measurements are discussed in this section.

Based on classical models that take into account the velocity gradient inside the probe volume (Durst, Melling and Whitelaw [9]), an analytical expression of the bias measurements is presented here, which will be compared to our measurements in the upstream laminar boundary layer.

If we consider a LDA measurement volume of a diameter $\Phi$ at a position $y$, the velocity measured at that 


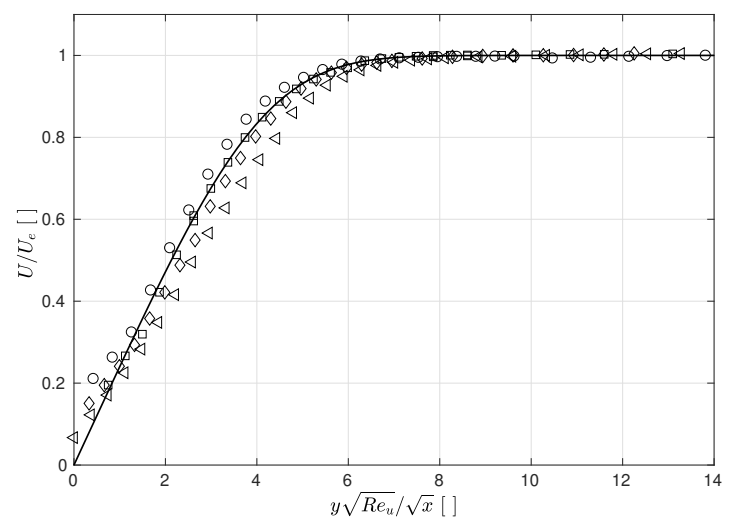

Fig. 5 Velocity profiles in normalised representation - LDA measurements From [8]. Line: laminar compressible boundary layer profile, $\circ: R e_{x}=4.48 \times 10^{5}, \square: R e_{x}=5.60 \times 10^{5}$, $\diamond: \operatorname{Re}_{x}=7.41 \times 10^{5}, \triangleleft: \operatorname{Re}_{x}=9.19 \times 10^{5}$.

position (at the center of the volume) is $U(y)$. At a position $\eta$ inside the measurement volume, $\left(-\frac{\Phi}{2}<\eta<\right.$ $\left.+\frac{\Phi}{2}\right)$, the associated velocity is, since the velocity profile is assumed to be linear :

$U(\eta)=U(y)+\alpha * \eta$

We assume that particles have the same transit probability through the measurement volume. This assumption could be not entirely true due to the non-uniformity of the seeding across the boundary layer, but is quite reasonable for the purpose of this paper, as it will be expanded hereafter. The probability $p(\eta)$ that a particle will be at a position $\eta$ when passing through the measurement volume of diameter $\Phi$ is $p(\eta)=\frac{1}{\Phi}$ so that $\int_{-\frac{\Phi}{2}}^{\frac{\Phi}{2}} p(\eta) d \eta=1$.

Then, the mean velocity $\overline{U_{\eta}}$ inside the measurement volume is obtained by:

$\overline{U_{\eta}}=\int_{-\frac{\Phi}{2}}^{\frac{\Phi}{2}} p(\eta) U(\eta) d \eta$

as well as the mean square velocity:

$\overline{U_{\eta}^{2}}=\int_{-\frac{\Phi}{2}}^{\frac{\Phi}{2}} p(\eta) U(\eta)^{2} d \eta$

From equation 3:

$\overline{U_{\eta}}=U_{y}$

This shows that the mean velocity is not biased due to the gradient effect. The second order moment can be derived from 4 :

$\overline{U_{\eta}^{2}}=U_{y}^{2}+\alpha^{2} \frac{\Phi^{2}}{12}$
The apparent velocity variance is defined as:

$\epsilon_{\alpha}^{2}=\overline{u^{\prime 2}}=\overline{U_{\eta}^{2}}-{\overline{U_{\eta}}}^{2}=\alpha^{2} \frac{\Phi^{2}}{12}$

In the initial part of the laminar boundary layer $U_{y}=$ $\alpha * y$, and $\alpha \simeq U_{e} /(0.7 \delta)$, the apparent rms velocity fluctuations are:

$\epsilon_{\alpha}=\frac{\alpha}{2 \sqrt{3}} \Phi \simeq \frac{U_{e}}{2 \sqrt{3}} \frac{\Phi}{0.7 \delta}$

We see that the apparent RMS velocity fluctuations measured by LDA in the gradient depend on the ratio $\Phi / \delta$. However, inside the interaction, the linear approximation for the mean velocity profile across the boundary layer is no longer relevant: the flow is strongly distorted in the recirculating region. Therefore, the velocity gradient inside the probe volume has to be estimated for each measurement point. It can be expressed as followed, with the subscript $i$ designating the current position of the measurement volume and $U_{i}$ the velocity at the position $i$.

$|\alpha|=\frac{1}{2}\left(\left|\frac{U_{i}-U_{i-1}}{y_{i}-y_{i-1}}\right|+\left|\frac{U_{i+1}-U_{i}}{y_{i+1}-y_{i}}\right|\right)$

This implies :

$\epsilon_{\alpha}=\frac{\Phi}{2 \sqrt{3}} * \frac{1}{2}\left(\left|\frac{U_{i}-U_{i-1}}{y_{i}-y_{i-1}}\right|+\left|\frac{U_{i+1}-U_{i}}{y_{i+1}-y_{i}}\right|\right)$

Of course this model is made according to a mean velocity profile which is linear, at least inside the measurement volume. For the measurement volumes under consideration, this is a quite reasonable assumption. The model depends also on the particular Probability Density Function of the position of the particles inside the probe volume. Nevertheless, using other PDF does not change significantly the results. For example, a Gaussian PDF with a standard deviation $\sigma$ defined as $2 p \sigma=\Phi$ can be used to describe the probability for validating a particle. The parameter $p$ defines the efficient spatial extent of validation for the particles compared with the probe volume diameter. Such PDF leads to the following expression:

$\epsilon_{\alpha}=\frac{\Phi}{2 p \sqrt{2}} * \frac{1}{2}\left(\left|\frac{U_{i}-U_{i-1}}{y_{i}-y_{i-1}}\right|+\left|\frac{U_{i+1}-U_{i}}{y_{i+1}-y_{i}}\right|\right)$

Equation 6 and 7 show that the bias remains directly proportional to the diameter of the probe volume. The diameter of the probe volume $\Phi$ and the fringes spacing $\delta_{f}$ are given by the relations (1). Note that the volume diameter is much more sensitive to the beam waist than to the angle between the two beams. Therefore, the beam expansion factor is the most efficient parameter to minimise the probe volume diameter. 
Table 1 LDA characteristics

\begin{tabular}{|c|l|l|l|l|l|}
\hline$\Phi[\mu m]$ & $\begin{array}{l}\text { Beam } \\
\text { spac- } \\
\text { ing } \\
{[\mathrm{mm}]}\end{array}$ & $\begin{array}{l}\text { Beam } \\
\text { ex- } \\
\text { pander } \\
\text { ratio }\end{array}$ & $\begin{array}{l}l \\
{[\mathrm{~mm}]}\end{array}$ & $\begin{array}{l}\text { Fringe } \\
\text { spac- } \\
\text { ing } \\
{[\mu m]}\end{array}$ & $\begin{array}{l}F_{\max } \\
{[\mathrm{MHz}]}\end{array}$ \\
\hline 75 & 19.2 & 1.98 & 1.9 & 6.70 & 110 \\
\hline 38 & 11 & 3.92 & 0.66 & 5.97 & 117 \\
\hline
\end{tabular}

When defining a probe volume for LDA measurements in high velocity flows, using a Bragg cell to resolve negatives velocities, one also has to take also into account the limited bandwidth of the electronic devices as well as the low signal intensity, due to the very short transit time and the small particle diameter. The photomultiplier's bandwidth is limited to about $180 \mathrm{MHz}$ and, to avoid any signal attenuation, the desired maximum Doppler frequency has been fixed at around $f_{D_{\max }}=$ $100-120 \mathrm{MHz}$. Taking into account the external velocity of the flow $\left(U_{e}=450 \mathrm{~ms}^{-1}\right)$ and the frequency shift of the Bragg Cell $\left(f_{\text {Bragg }}=40 M \mathrm{Mz}\right)$, this defines the shortest inter-fringe which can be used: $\delta_{f_{\text {min }}}=$ $U_{e} /\left(f_{D \max }-f_{\text {Bragg }}\right)=5.6-7.5 \mu \mathrm{m}$.

The LDA configuration with a reduced probe volume is derived from the $75 \mu \mathrm{m}$ one. Firstly, the adjustable beam spacer has been used: the distance between beams has been fixed at $11 \mathrm{~mm}$. Then, two beam expanders are used: each beam expander has a beam expansion coefficient of 1.98 , the resulting coefficient is thus 3.92 . The same coefficient is applied on the final beam spacing. Finally, a $500 \mathrm{~mm}$ converging lens is used, leading to a measurement volume of a diameter of $38 \mu \mathrm{m}$ and a length of $0.656 \mathrm{~mm}$. Main parameters of the $75 \mu \mathrm{m}$ and $38 \mu \mathrm{m}$ LDA settings are reported in Table 1. For both set-ups, the fringe spacing is maintained nearly constant, at around $7 \mu \mathrm{m}$, corresponding to a maximum Doppler frequency of about $110 \mathrm{MHz}$, as expected. Of course, the number of fringes is nearly divided by two in the case of the $38 \mu \mathrm{m}$ probe volume. This will proportionally increase the uncertainty on the Doppler frequency evaluation: this point will be expanded later.

In a standard LDA configuration, the receiving optic would normally have the same optical configuration as for the emitting optic. Here, only one beam expander is used. Thus, due to the asymmetry of the optical system, the magnification of the probe volume is reduced and, since we are in an off-axis configuration, the probe volume has an apparent length long enough to optimise the data rate. The $38 \mu m$ LDA set-up is shown in Figure 6.

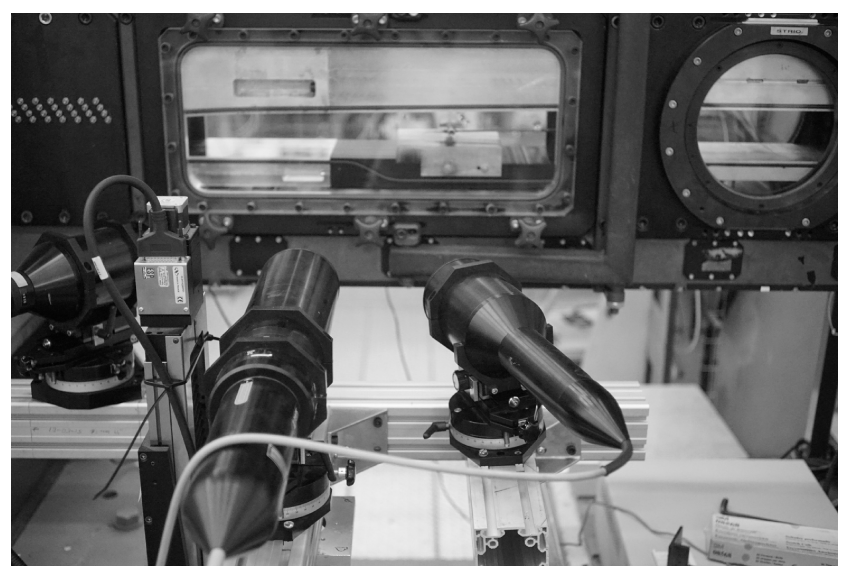

Fig. $638 \mu m$ LDA set-up

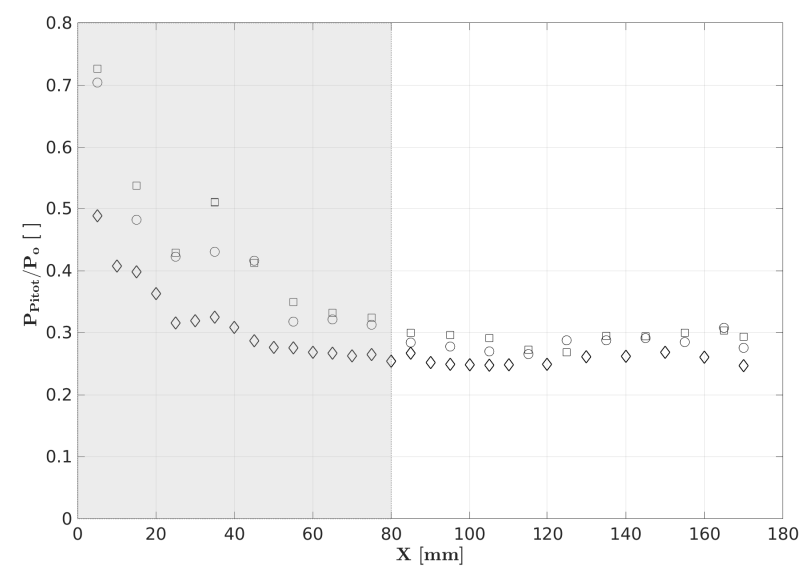

Fig. 7 Evolution of total pressure ratio along the flat plate. The gray zone indicates the part of the flow where the sensor is larger than the boundary layer. $\diamond: P_{0}=0.20 \mathrm{~atm}, \circ: P_{0}=$ $0.20 \mathrm{~atm}, \square: P_{0}=0.75 \mathrm{~atm}$

\section{Upstream laminar boundary layer}

The boundary layer which is developing along the flat plate has been determined to be laminar. The transition of a thin boundary layer from laminar to turbulent can be detected by means of total pressure measurements at the wall. In such configuration, Pitot measurements are indicative: the Pitot probe is far too large to expect spatially accurate measurements. Nevertheless, we know that such a probe placed at the wall will give measurements proportional to the wall friction and the velocity shape and thus is a good indicator of the turbulent state: it is possible to detect the transition location based on these measurements by observing a change in the value of the pressure ratio [23].As for an example, in the same facility, a factor of two has been observed along the transitional region of a Mach 2.3 boundary layer. 
Total pressure measurements have been made in the boundary layer, using a flattened pitot probe: the thickness of the probe is $0.3 \mathrm{~mm}$, and the pressure tap is $0.1 \mathrm{~mm}$. It means that when the pitot probe is placed at the wall, the first points of total pressure measurement are at $0.15 \mathrm{~mm}$ in height, with a spatial integration from $0.1 \mathrm{~mm}$ to $0.2 \mathrm{~mm}$. Consequently, since the boundary layer thickness is very small in the first centimeters of its development, measurements will have no significance.

Figure 7 presents the total pressure measurements along the flat plate, for several stagnation pressures from $0.15 \mathrm{~atm}$ to $0.75 \mathrm{~atm}$. They are normalised with the stagnation pressure of the flow $P_{0}$. In the first $80 \mathrm{~mm}$, the probe size is too large and the total pressure is decreasing as long as the boundary layer remains thinner than the probe. Nevertheless, we see that up to $175 \mathrm{~mm}$ (at the end of the flat plate), no transition effects are detectable, since no increase of pressure ratio is observed. It can be concluded from these measurements that the boundary layer is laminar all over the flat plate, up to $P_{0}=0.75 \mathrm{~atm}$, equivalent to a Reynolds number of $R e_{x}=1.85 \times 10^{6}$.

These results have been confirmed with more quantitative measurements, using a Laser Doppler Anemometer (LDA) [8]). As shown in the previous section, mean velocity profiles are unbiased. The laminar state of the boundary layer was investigated by comparing mean velocity profiles to a compressible laminar profile obtained by numerical simulation. For a theoretical laminar boundary layer, velocity profiles are self-similar: $U / U e=f\left(y \sqrt{R e_{u}} / \sqrt{x}\right)$, where $\sqrt{R e_{u}}$ is the unit Reynolds number [4]. The boundary layer thickness evolves as :

$$
\delta \sim \frac{x}{\sqrt{R e_{x}}}
$$

Figure 5 represents the mean velocity profiles obtained by LDA using the $75 \mu \mathrm{m}$ probe volume, for positions ranging from $80 \mathrm{~mm}$ to $160 \mathrm{~mm}$ and for stagnation pressures from $0.4 \mathrm{~atm}$ to $0.8 \mathrm{~atm}$, leading to Reynolds numbers $R e_{x}$ from $0.448 \times 10^{6}$ to $0.919 \times 10^{6}$. As seen on the figure, boundary layer profiles are in very good agreement with a simulated compressible boundary layer profile. From the similarity obtained for the mean longitudinal velocity profiles, dimensionless boundary layer thicknesses can be derived, with a confidence interval of about $\pm 5 \%$ :

$\bar{\delta}=\delta \sqrt{R e_{u}} / \sqrt{x}=6.67$

and

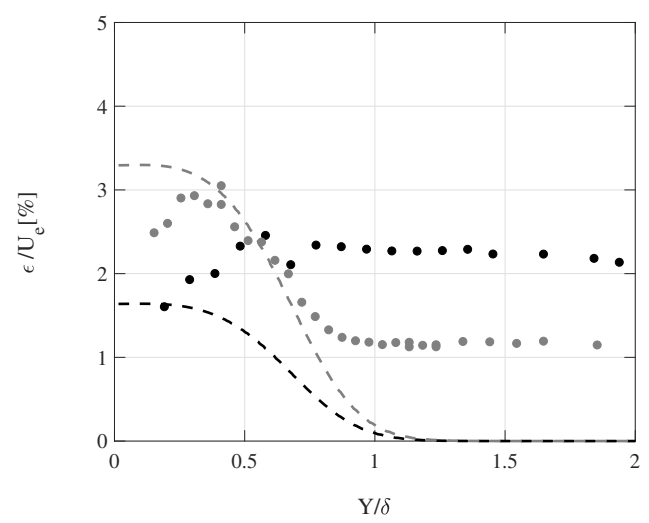

Fig. 8 Biased turbulence intensity as measured in the upstream boundary (symbols), modeling of the probe volume integration effects (- -) see Eq.5. The diameter $75 \mu \mathrm{m}$ is in gray and the $38 \mu m$ in black.

$$
\begin{aligned}
& \overline{\delta_{i 1}^{*}}=\delta_{i 1}^{*} \sqrt{R e_{u}} / \sqrt{x}=2374 \\
& \overline{\delta_{i 2}^{*}}=\delta_{i 2}^{*} \sqrt{R e_{u}} / \sqrt{x}=908
\end{aligned}
$$

Thus, the incompressible shape factor of the velocity profiles is 2.61. Note that the value of $\bar{\delta}$ can be estimated from Cousteix [4] formula. The derived value is 7.38 , about $10 \%$ larger than the present results. The slight difference with the theoretical profile of the velocity measurements may be related to the accuracy of the vertical position. For example, with a stagnation pressure of $0.75 \mathrm{~atm}$ and at a measurement position $x=100 \mathrm{~mm}$, a correction of $0.05 \mathrm{~mm}$ on the $y$ position corresponds to a correction of 0.52 on the quantity $y \sqrt{R e_{u}} / \sqrt{x}$.

The models that are to take into account the noise of the LDA measurements (eq.5 or eq.6) was tested in the upstream laminar boundary layer where the velocity fluctuations are negligible with respect to the LDA measurement. In the external flow, where the velocity gradient is vanishing, the amplitude of the velocity fluctuations is lower than the expected LDA resolution and the free-stream measurements can be considered as a measurement of the Doppler frequency uncertainty.

The standard deviation of the longitudinal velocity measured across the boundary layer is reported in Figure 8 for the two different probe volumes $75 \mu \mathrm{m}$ and $38 \mu \mathrm{m}$, respectively in gray and black. Superimposed are the apparent turbulent intensities derived from the model given in Eq.6. Two results can be derived from this figure:

- the model does not describe accurately the measured turbulence intensity for the two probe volumes 
- the noise from the Fourier analysis of the Doppler bursts (about 1 and $2 \%$ outside of the shear region for respectively the $75 \mu \mathrm{m}$ and the $38 \mu \mathrm{m}$ configurations) is of the same order of magnitude as the modeled turbulent intensity and becomes dominant near the edge of the boundary layer. Therefore, it must also be taken into account.

The uncertainty for the Doppler frequency $f_{D}$ is proportional to $k / \Delta T$, where $\Delta T=\Phi / U$ is the transit time of the particle and $k$ a coefficient depending on the Fourier interpolation process used in the BSA processor. In the external flow, the transit time depends only on the probe volume diameter $\left(\Delta T=\Phi / U_{e}\right)$ and the Doppler velocity uncertainty is $\epsilon_{f}=k \delta_{f} / \Delta T$ where $\delta_{f}$ is the interfringe. Therefore, the coefficient $k$ can be estimated from the measurements achieved for $y \gg \delta$ and the velocity uncertainty across the boundary layer is:

$\epsilon_{f}=k \delta_{f} U / \Phi$

The estimated value for the coefficient $k$ was 0.1 , about a tenth of the discrete Fourier analysis.

Finally, considering that the Doppler and the gradient velocity uncertainties are independent random variables, the variance of overall noise for the LDA measurements is $\epsilon^{2}=\epsilon_{\alpha}^{2}+\epsilon_{f}^{2}$, where $\epsilon_{\alpha}$ is estimated from Eq.6. Results are presented in Figure 9. The same colors and symbols as in Figure 8 are used. The numerical mean velocity profile (see Figure 5) is also reported.

From Figure 9, it is clear that the model is adequate to describe the LDA measurement uncertainty across the boundary layer. In particular, typical shape of the overall noise is adequately reproduced. This final shape derives from the combination of gradient noise (see equation 6) and Fourrier noise (see equation 8). The Fourrier noise is intrinsic and does not depend on any particular hypothesis. Only the $k$ factor, which depends on BSA (Burst Spectrum Analyser) post-processing, is adjusted using external measurements. As expected, it has been found constant for both measurements volumes $(k=0.1)$. The gradient noise, as already mentioned, depends on the probability density function of the particle position inside the probe volume. Nevertheless, whatever the specific Probability Density Function considered, an equivalent uniform Probability Density Function can be defined by adjusting an effective measurement probe volume diameter (defined as $\Phi / p$, see equation 7).

The $38 \mu m$ set-up, which exhibits the largest noise in the external flow, is more efficient for achievieng unsteady measurements inside the shear layer region $(y / \delta<0.6)$ and has been used to document the interaction region

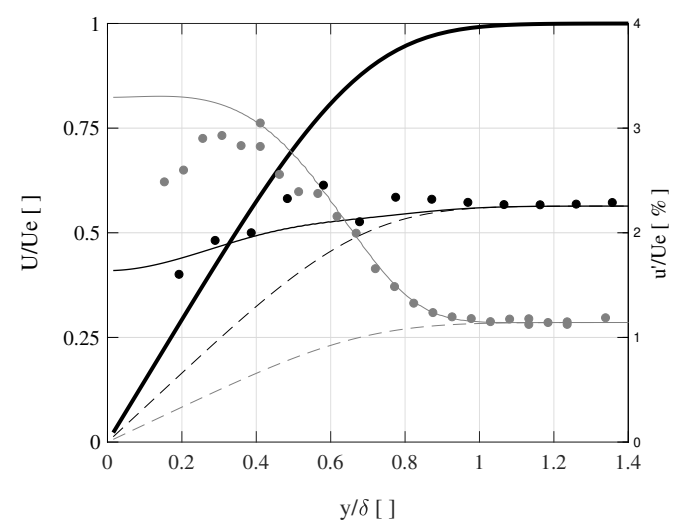

Fig. 9 Turbulence intensity measured in the upstream boundary layer (symbols), modeling of the probe volume integration effects (-) see Eq.5 and of the Fourier uncertainties $(--)$ see Eq.8. The diameter $75 \mu \mathrm{m}$ is in gray and the $38 \mu \mathrm{m}$ in black.

where larger velocity fluctuations are expected. Nevertheless, it is clear that, whatever the LDA set-up, no significant unsteady measurements can be achieved in the laminar boundary layer: the expected levels are too small when compared with the artificial turbulence or with the remaining noise inherent to the measuring system.

These results will be used to define the regions of interaction where significant unsteady measurements can be achieved: turbulence levels have to be larger than the overall noise modeled from the unbiased mean velocity profiles.

\section{Shock Wave Boundary Layer Interaction}

\subsection{Longitudinal development of the interaction}

Figures 10(a), 10(b) and 11 present the mean and turbulent longitudinal velocity measurements at several sections along the interaction, achieved with the $38 \mu \mathrm{m}$ probe volume LDA anemometer.

The longitudinal mean velocity profiles evolve gradually from a laminar to inflectional profiles in the interaction region, then to filled velocity profiles downstream from the reattachment point. In the initial interaction region, near-wall points had very low data rate and measurements were not reliable.

A compilation of the mean longitudinal velocity profiles are reported in Figure 10(a) for several sections along the interaction. Additionally, the boundary layer thickness as well as a first approximation of the recirculated zone are represented. This last quantity corresponds to the location of the first positive velocity measured. 


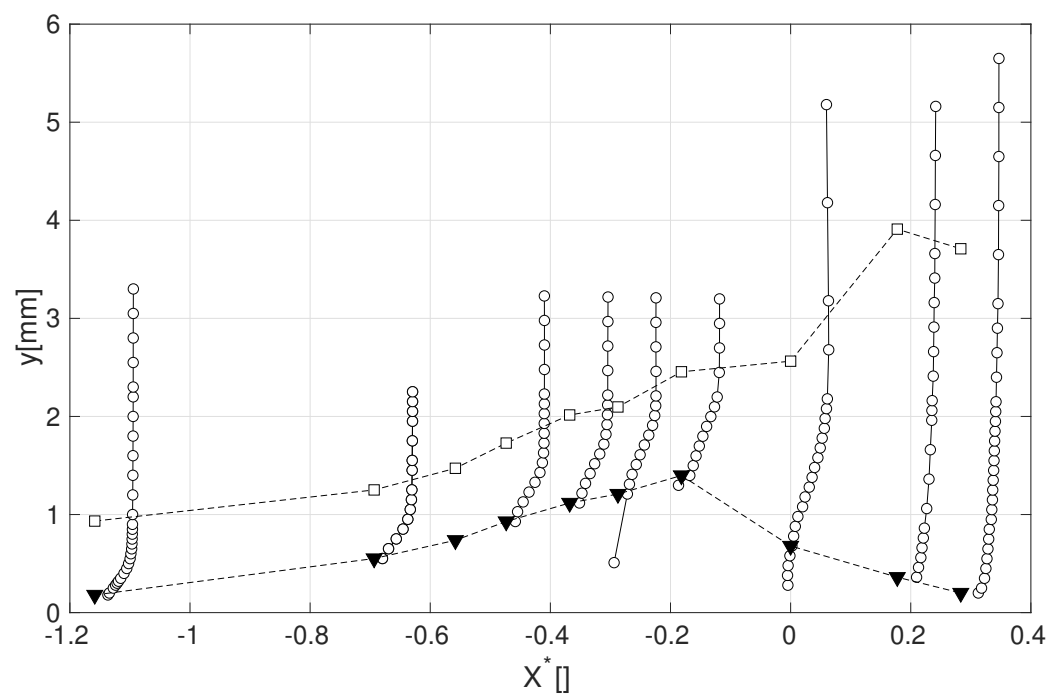

(a)

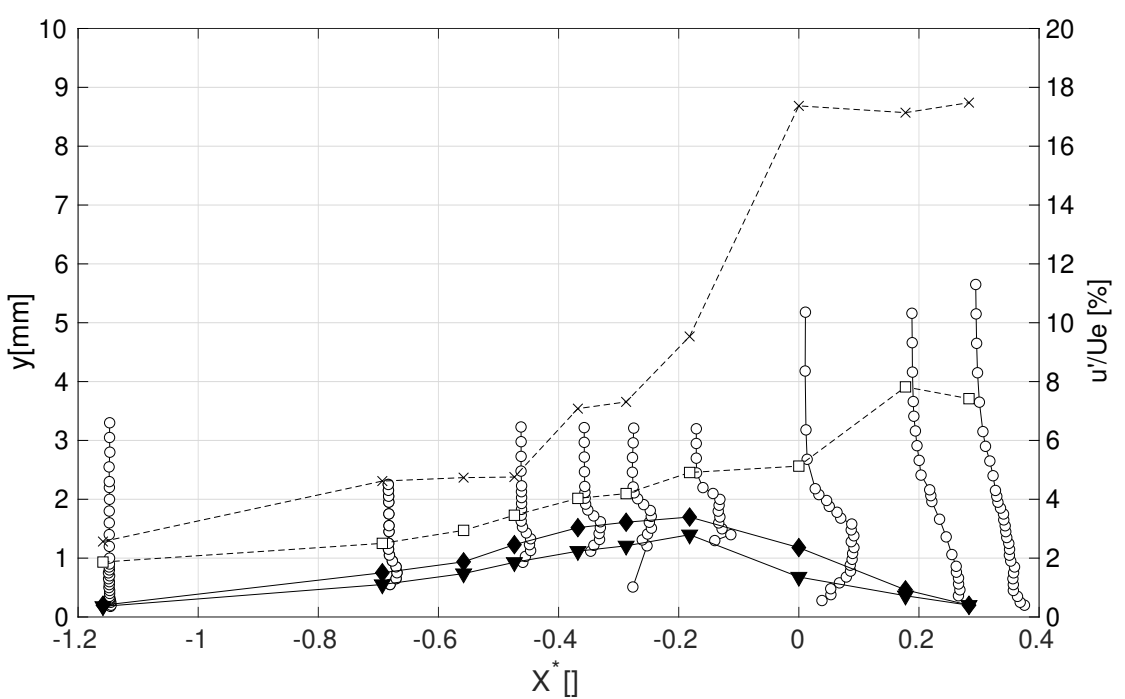

(b)

Fig. 10 Mean (a) and turbulent (b) longitudinal velocity LDA measurements along the interaction with the $38 \mu m$ probe volume, $P_{0}=0.4 \mathrm{~atm} . \circ: \mathrm{LDA}$ measurements, $\boldsymbol{\nabla}$ : first positive velocity measured, $\square: \delta_{99}$, : position of the maximum of turbulence intensity, $\times$ : maximum turbulence intensity (right axis scale, figure (b) ). Position of profiles, from left to right: $X^{*}=[-1.26 ;-0.74 ;-0.59 ;-0.5 ;-0.38 ;-0.18 ; 0.02 ; 0.22 ; 0.34]$.

As already mentioned, in the first part of the recirculation zone, no - or very few- particles are present, and this line (triangles) is located at the last position where measurements were possible. Its longitudinal elevation can be considered as an approximation of the streamline corresponding to the unseeded region. Downstream from the station $X^{*}=-0.18$, the turbulence intensity of velocity fluctuations is increasing dramatically (see Figure 10(b)): the shear layer is seeded down to the wall and the mean recirculating region is clearly resolved by the LDA measurements (see Fig- ure 11(e)). Flow is reattaching between the locations $X^{*}=0$ and $X^{*}=0.2$.

The apex (maximum elevation of the separated bubble) is found at $X^{*}=-0.2$ at $y=1.3 \mathrm{~mm}$. As the length of interaction is $43.7 \mathrm{~mm}$, this leads to a flow deviation at the separation point of about $\theta_{s e p}=\tan ^{-1}(1.3 /(0.8 *$ $L)) \simeq 2.1$ degrees.

The turbulence intensities $\sqrt{\overline{u^{\prime 2}} / U_{e}}$ are shown in Figure 11, together with the artificial turbulence estimated from relation 6. As shown in the previous section, the unsteady measurements (RMS velocity) in the upstream 


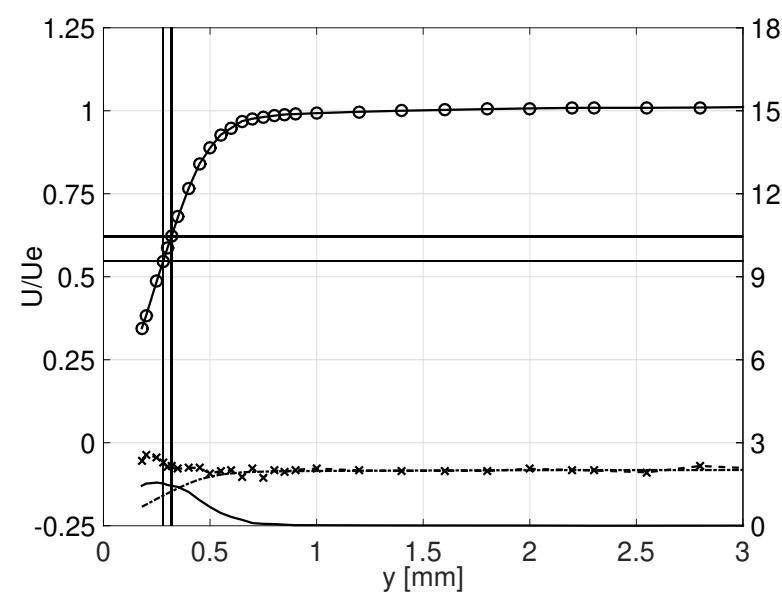

(a)

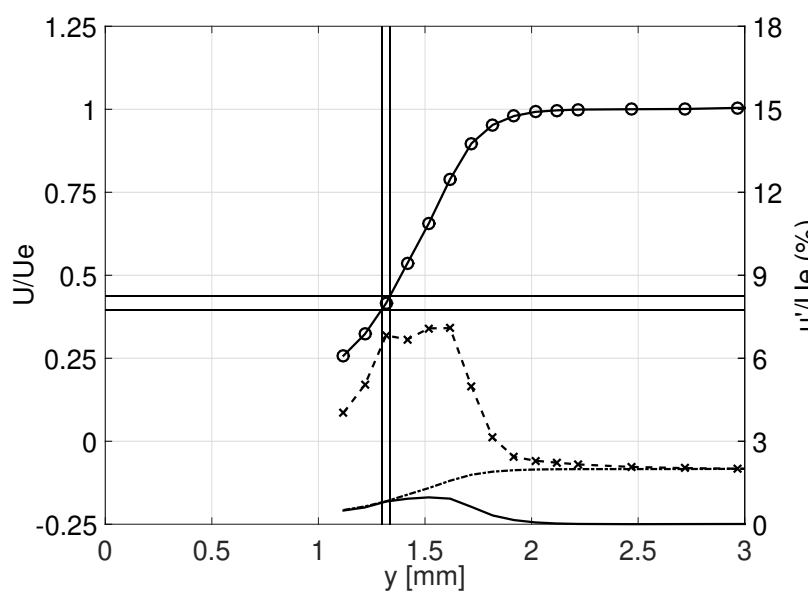

(c)

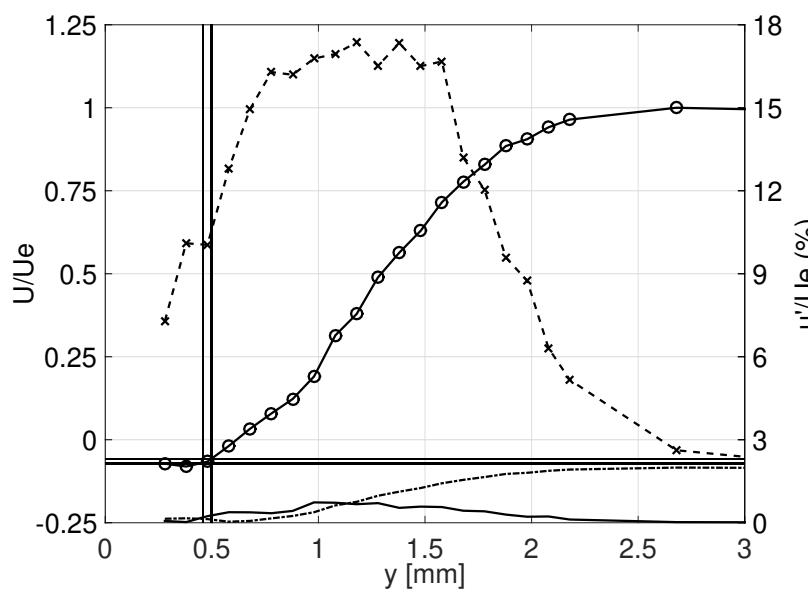

(e)

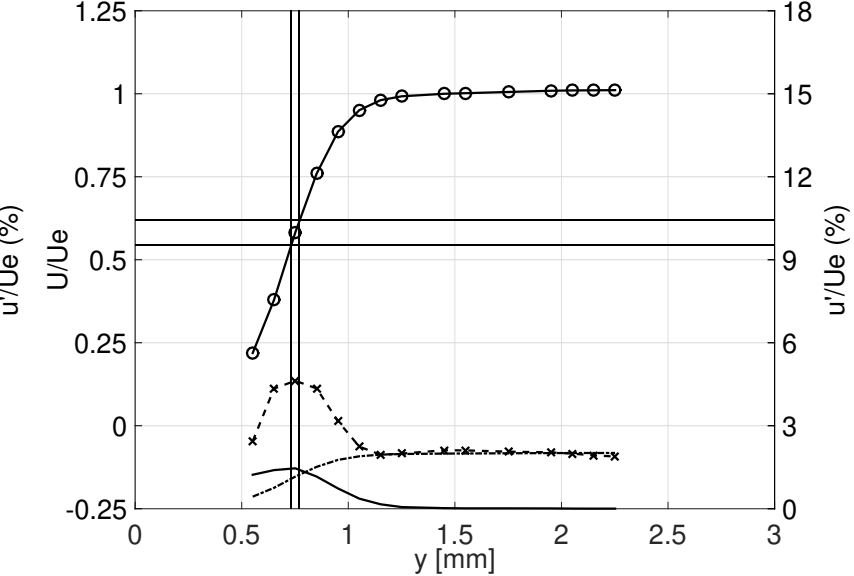

(b)

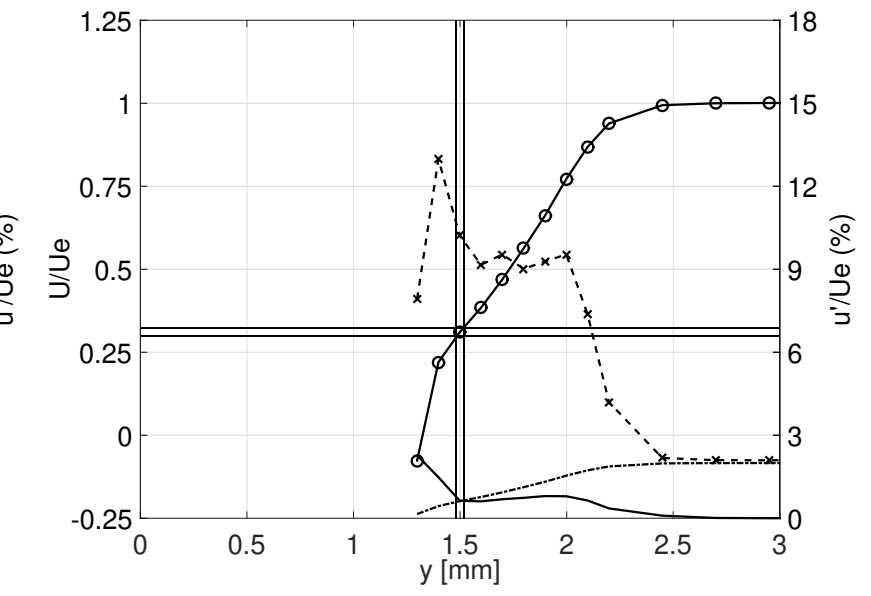

(d)

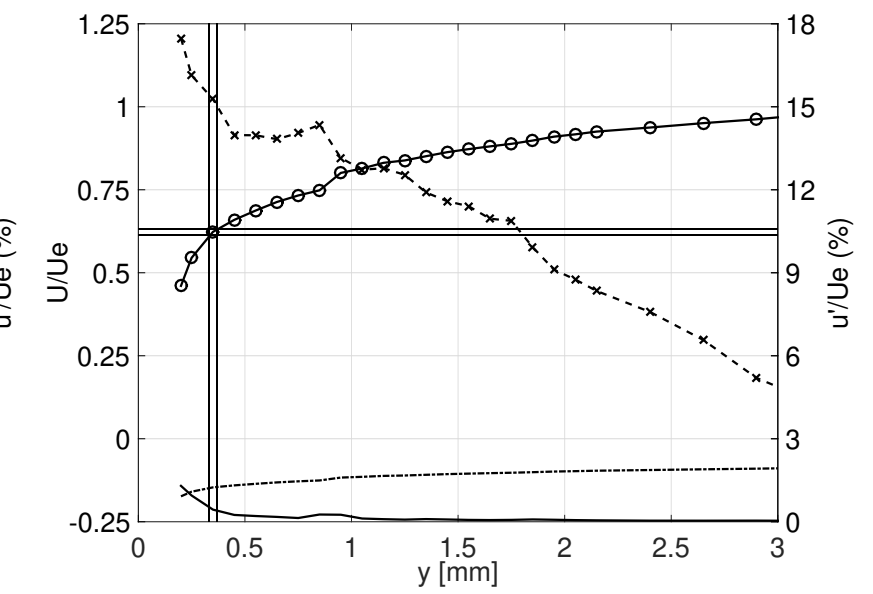

(f)

Fig. 11 Mean and turbulent longitudinal velocity obtained by LDA, $P_{0}=0.4 a t m$ : (a) $X^{*}=-1.26,(\mathrm{~b}) X^{*}=-0.74$, (c) $X^{*}=-0.38$, (d) $X^{*}=-0.18$, (e) $X^{*}=0.02$, (f) $X^{*}=0.34$. - : LDA measurements, $*$ : velocity fluctuations, $-:$ artificial turbulence, -. : Fourier uncertainties 
laminar boundary layer are not significant (see Figure 11(a)). However, the fluctuations that are developing along the mixing layer region over the separated bubble, are significantly larger than the artificial turbulence reported on the figure: about twice as large near the separation $\left(X^{*}=-0.74\right)$ and an order of magnitude near the impingement location. This shows that, taking into account the artificial turbulence estimated from the mean velocity profile, quite accurate turbulent measurements can be achieved within the interaction. In fact, using a reduced probe volume tends to increase the signal to noise ratio (SNR). Considering, for example, Figure 11(b), the maximum measured level of velocty fluctuation, at $y=0.7 \mathrm{~mm}$ is of $4.6 \%$. In this configuration the model predicts that an artificial turbulence with the $38 \mu \mathrm{m}$ probe volume diameter is of $2 \%$ as well as the FFT noise.

The velocity variance measured $\sigma_{m}^{2}$ is then $\sigma_{m}^{2}=$ $\epsilon_{\alpha}^{2}+\epsilon_{f}^{2}+\sigma_{u, \text { real }}^{2}$, where $\sigma_{u, \text { real }}^{2}$ is the real variance of the velocity fluctuations. The real standard deviation is of the order of $3.6 \%$. The calculation of the artificial turbulence generated by the velocity gradient at this point with a $75 \mu \mathrm{m}$ probe volume diameter gives a value of $4.1 \%$, and the fft noise of $\approx 1 \%$. This means that the measured turbulence intensity would have been $5.6 \%$. Thus, the SNR is 1.28 for the reduced probe volume, and 0.86 for the classical one. Confidence in measurements, in flow regions where the turbulence intensity is quite reduced, is then strengthened. In studying the transition flow process in such interactions, this gain on SNR is of fundamental importance. Downstream from the separation point, typical profiles of mixing layers can be observed. The maximum RMS of the velocity fluctuations is increasing along the interaction: their location and amplitudes are reported in Figure 10(b). Their amplitude is found to increase from about $2 \%$ of the external upstream velocity - which is the noise level of these LDA measurements - to a saturation level of about $0.17 U_{e}$ near the incident shock impingement location. The velocity profiles measured at $X^{*}=0.35$, downstream of the interaction, clearly show that the boundary layer is no longer laminar, see Figure 11(f). The incompressible shape factor at this position is $H_{i}=$ 1.62 , similar to turbulent profiles. It has to be mentionned that it was calculated by integrating the velocity profile, without any shape fitting procedure as suggested in [29].

In order to qualify the turbulent state of the boundary layer downstream of the reattachment, Figure 12(a) presents the Van Driest representation of mean velocity profiles downstream of the interaction. No well-defined log-law region can be observed: the boundary layer is not in a classical turbulent state. Velocity fluctuations

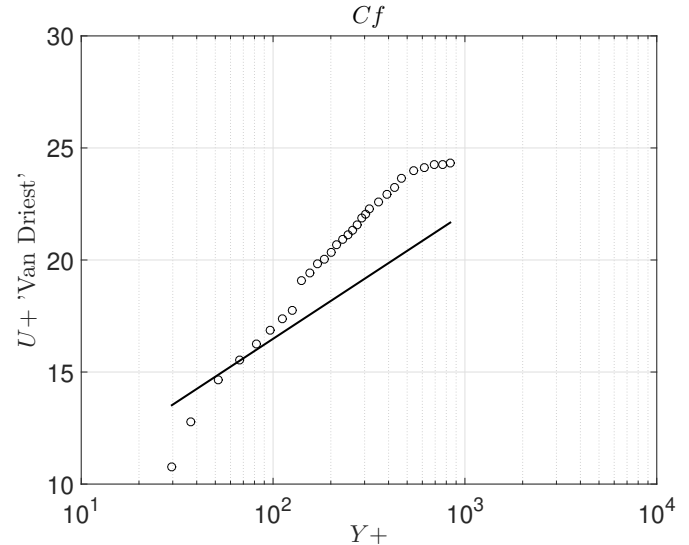

(a)

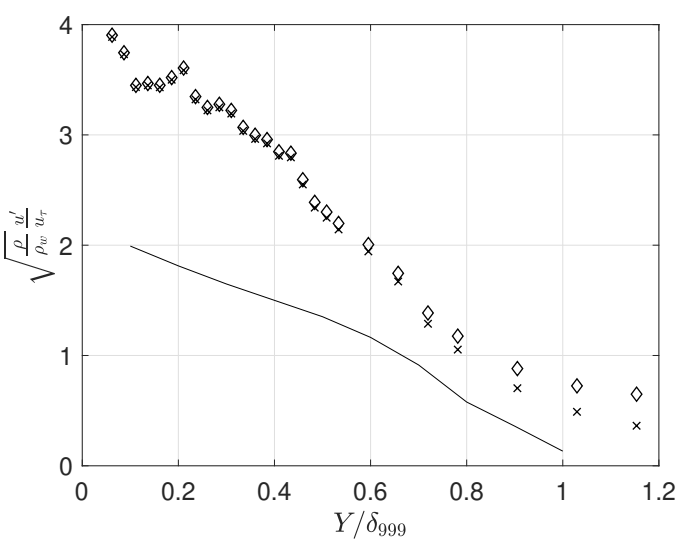

(b)

Fig. 12 Van Driest (a) and Morkovin (b) representation of the velocity profile downstream of interaction the interaction $\left(X^{*}=0.34\right), P_{0}=0.4 a t m . \circ:$ Mean velocity, $\diamond:$ Measured velocity fluctuations, $\times$ : corrected velocity fluctuations

are shown in Figure 12(b) together with classical incompressible turbulent profiles [14]. Large velocity fluctuations are observed, higher than expected for classical turbulent profiles: at this position, about 30 displacement thickness downstream from the impingement location $\left(\simeq 30 \delta_{i m p}^{*}\right)$ the flow is still in the relaxing part of the interaction.

\subsection{Transitional mechanism along the interaction}

From the results reported in Figures 10(a) to 12 a global overview of the transition process along the interaction can be inferred. This is reported in Figure 13. The upstream flow is a laminar boundary layer with a quasi linear velocity profile up to $U / U_{e} \simeq 0.6$. At the separation point $\left(X^{*}=-1\right)$, compression waves are developing and the decelerated shear layer is growing down 


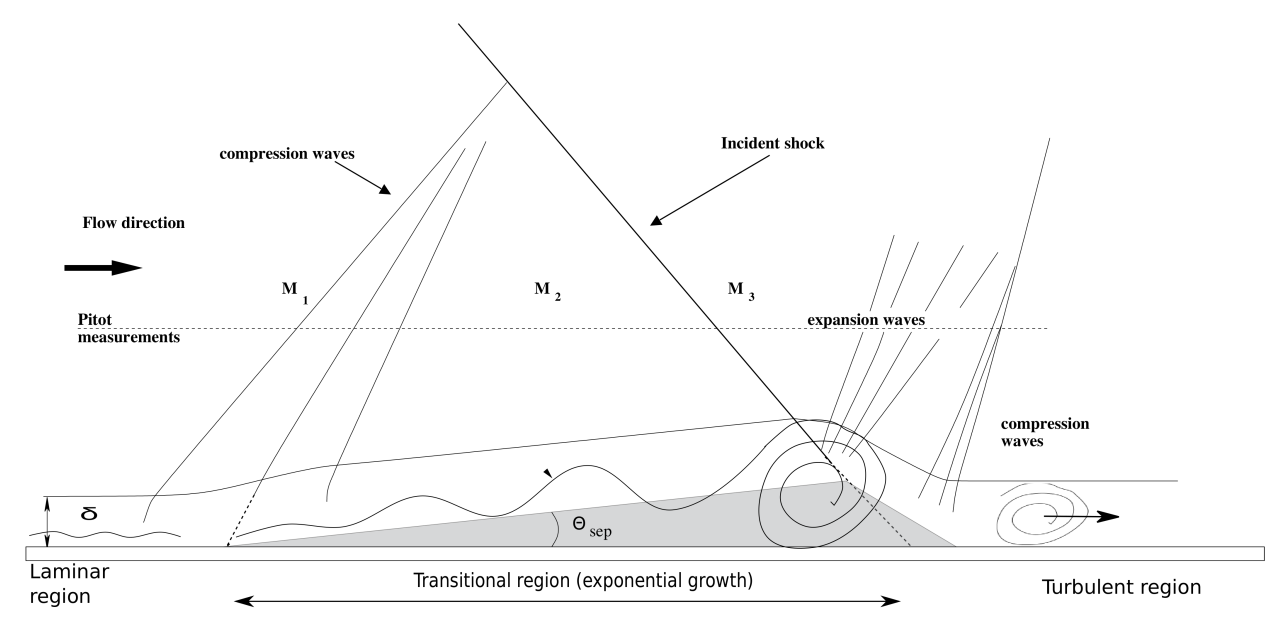

Fig. 13 Sketch of the transition development along the interaction.

to the apex location, around $X^{*}=-0.2$. The longitudinal evolution of the location of the velocity fluctuations maxima are reported Figure 14. A linear best fit of the point inside the interaction $\left(0.2<X^{*}<0.8\right)$ has given a slope of 1.872 (the $95 \%$ confidence bounds are 1.344 and 2.399). This corresponds to a deviation angle of 2.45 degrees (respectively 1.8 degrees to 3.1 degrees), which is consistent with the mean velocity measurements. The flow deviation across compression waves near the separation point was estimated from Pitot measurements (see Figure 4): a deviation of 2 degrees was evaluated. This corresponds to only the half of the imposed flow deviation. From classical Free Interaction Theory [3], the plateau pressure of the interaction can be estimated as:

$$
\frac{p-p_{0}}{p_{0}}=\frac{1}{2} \gamma M_{0}^{2} F \sqrt{\frac{2 C_{f 0}}{\left(M_{0}^{2}-1\right)^{1 / 2}}}
$$

where the index 0 refers to the upstream conditions and $F=1.5$ for laminar conditions. For the present experimental conditions, the pressure $p$ calculated from this relation corresponds to the pressure behind an oblique shock wave associated with a flow deviation of 2.2 degrees. The theoretical slope is reported on Figure 14. Taking into account the experimental uncertainties for such conditions, these different estimations are in good agreement.

Downstream from the separation point, typical profiles of mixing layer can be observed, with reverse flow of about $8.5 \%$ of the upstream external velocity. In the initial part of the interaction $\left(-1<X^{*}<-0.6\right)$, the amplitude of the velocity of fluctuations cannot be resolved from the present LDA measurements. In the second part $\left(-0.6<X^{*}\right)$, their amplitude can be resolved accurately and it is found to increase exponentially.

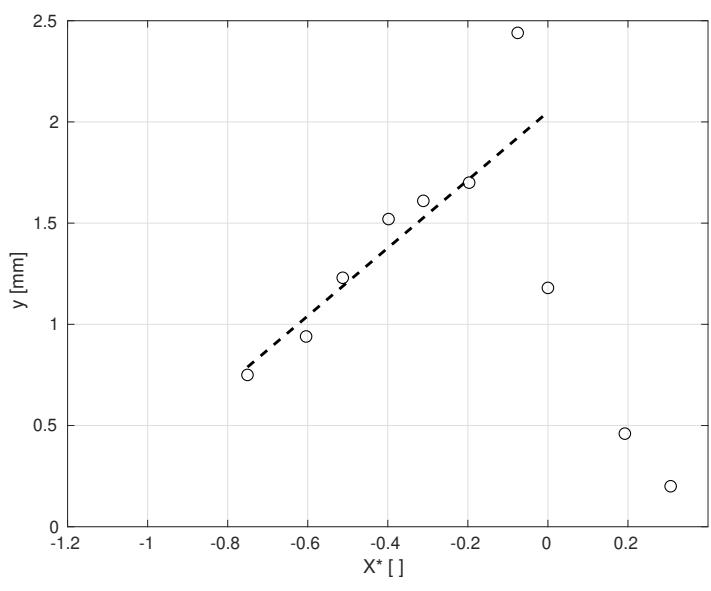

Fig. 14 Longitudinal evolution of the elevation of the maxima of RMS velocity along the interaction. Dashed line corresponds to the flow deviation estimated from Eq.9

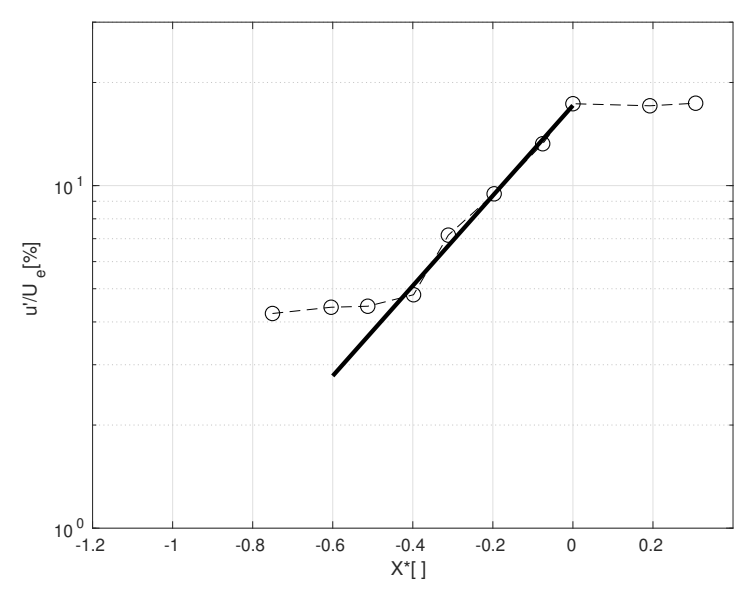

Fig. 15 Longitudinal evolution of the maxima of RMS along the interaction. Black line : exponential law $\left(17.14 \times e^{3.03 X^{*}}\right)$. 
This is compatible with the development of unsteady modes following a linear mechanism [28]. An exponential best fit of the data in the second part of the interaction is reported Figure 15. It corresponds to the exponential law:

$\frac{u^{\prime}(X) / U_{e}}{u^{\prime}\left(X_{1}\right) / U_{e}}=e^{\left(b * X^{*}\right)}$

with $b=3.03$. Or

$\frac{u^{\prime}(X)}{u^{\prime}\left(X_{1}\right)}=e^{\left(G *\left(X-X_{1}\right)\right)}$

with $G=0.069 \mathrm{~mm}^{-1}$ and $u^{\prime}\left(X_{1}\right)=0.17 * U_{e}$, where the velocity fluctuations maximum is located at the section $X_{1}$. In this configuration, the section is close to the incident shock impingement $\left(X=X_{i m p}\right)$. Transition to turbulence is occurring in this region $\left(0<X^{*}<0.2\right)$. It increases significantly the momentum transfer across the shear layer, as shown from the large increase of seeding in the near-wall regions: this promotes the reattachment of the flow. The velocity fluctuations maxima is found to decrease slightly, and then to remain at a nearly constant level down to $X^{*}=0.34$.

At this station, near-wall logarithmic region of the boundary layer is not observed, as is expected if the transition to fully turbulent flow mechanism was completed. Nevertheless, the velocity profiles are already filled and the incompressible shape factor is similar to fully turbulent profiles. In the downstream flow, turbulent intensities are strongly amplified if compared to classical zero pressure gradient turbulent boundary layers (nearly multiplied by 2). This suggests that the unsteadiness that is developing along the separated shear layer is generating energetic structures in the vicinity of the apex which are shed downstream, as has been observed in separated Shock Wave Turbulent Boundary layer Interactions [24].

One of the main results of the present work is the possible relation between the amplification mechanisms of the unsteadiness inside the separated shear layer, and the location of the reattachement: the plateau level shows a saturation of the exponential amplification of the upstream perturbations along the separated shear layer. As this saturation region is found in the vicinity of reattachment region, it suggests that, for transitional interactions, the reattachment is set by the saturation of the amplification process of the velocity fluctuations. This leads to a very asymmetric bubble, unlike the fully laminar separations, where nearly symmetric separation bubbles are expected [6].

When the transition is nearly achieved, energetic structures are created and generate mixing across the shear layer. This significantly increases the momentum in the near-wall region causing reattachment. The mechanism of amplification of upstream perturbations along the separated shear layer can be expected to depend on the amplitude and/or the nature of the perturbations in the upstream laminar boundary layer. Unfortunately, measurements of these perturbations are beyond the possibilities of such LDA measurements: they involve amplitudes which are at least one order of magnitude lower than the effective resolution of the LDA anemometer. In the case of the IUSTI's supersonic wind tunnel, very large aspect ratios have been obtained $\left(L / \delta_{0 i}^{*}=174\right)$ for experiments realised at an impingement Reynolds number of $R e_{i m p}=6.04 \times 10^{5}$. This has to be compared with the aspect ratios found by [12], which was of $L / \delta_{0 i}^{*} \approx 100$ in the case of larger Reynolds number $\left(R e=1.9 \times 10^{6}\right)$ in a blow-down facility.

As the sensitivity of the transition mechanisms to the initial conditions (upstream perturbations, shock intensity, Reynolds and Mach number) is a major concern in such flows, several other experiments including unsteady measurements and covering as a wide range of parameters as possible would be of interest.

\section{Conclusions}

A transitional shock wave reflection has been documented for a Mach number of 1.68, at limited Reynolds number. The imposed flow deviation of 5 degrees generates a separated interaction, with a large aspect ratio $\left(L / \delta_{0 i}^{*}=174\right)$. The boundary thickness of the undisturbed laminar boundary layer at the impingement location is $0.9 \mathrm{~mm}$ and at its maximum elevation, the shear layer is only $1.3 \mathrm{~mm}$ thick. Therefore, a specific LDA set up has been defined, in order to obtain refined measurements and reduce as much as possible the integration effect across the probe volume. This makes it possible to minimise the diameter of the probe volume $(\Phi=38 \mu \mathrm{m})$ without too large Doppler frequencies. The effects on fluctuation measurements have been evaluated thanks to a simple stochastic model of the integration effects and have been compared with experimental results. If the mean velocity profiles can be accurately described, at least in regions where the flow is reasonably seeded, the RMS velocity fluctuations contain an apparent turbulence which depends on the local mean velocity and velocity gradient. Although no significant unsteady measurements are possible in the upstream laminar boundary layer due to fluctuation levels lower than the noise coming from the integration effects, accurate experimental results have been possible along the separated shear layer. Longitudinal evolution of the velocity fluctuations has been presented for a $5^{\circ}$ flow de- 
viation at a stagnation pressure of $0.4 a t m$. These show a longitudinal amplification of the velocity fluctuations with an exponential growth. These results are compatible with a linear amplification of perturbations along the interaction. The amplification process is thus attaining a plateau level, where the transition to turbulence dramatically increases the mixing across the shear layer, leading to the reattachment of the flow. Energetic eddies are shed downstream, and the relaxation process is still incomplete at 30 impingement boundary layer displacement thicknesses downstream. This mechanism will have to be validated for other configurations.

Acknowledgements This work received financial support by the European STREP TFAST (contract no. ACPO-GA2011-265455), and the project leading to this publication has received funding from Excellence Initiative of Aix-Marseille University - A*MIDEX, a French "Investissements d'Avenir" programme. It has been carried out in the framework of the Labex MEC. This support is gratefully acknowledged.

\section{References}

1. Ackeret, J., Feldmann, F., Rott, N.: Untersuchungen an Verdichtungsstossen und Grenzschichten in schnell bewegten Gasen. Mittelungen aus dem Institut für Aerodynamik 10 (1946)

2. Boutier, A.: Laser Velocimetry in Fluid Mechanics, pp. i-xiv. ISTE Ltd (2013). DOI 10.1002/9781118569610.fmatter

3. Chapman, D.R., Kuehn, D.M., Larson, H.K.: Investigation of separated flow in supersonic and subsonic streams with emphasis of the effect of transition. Tech. rep. (1957)

4. Cousteix, J.: Couche limite laminaire, cepadues edn. (1988)

5. Debiève, J.F., Dupont, P., Dussauge, J.P., Larchevêque, L., Piponniau, S.: Oblique shock reflection at $\mathrm{M}=1.6$ in situation of laminar/turbulent transition (experiment and LES). . Technical Report $\mathrm{N}^{\circ} 1.2 .9$ (2013)

6. Degrez, G., Boccadoro, C.H., Wendt, J.F.: The interaction of an oblique shock wave with a laminar boundary layer revisited. an experimental and numerical study. Journal of Fluid Mechanics 177, 247-263 (1987). DOI 10.1017/S0022112087000946

7. Délery, J.M., Marvin, J.G.: Shock wave - boundary layer interactions. Tech. rep. (1986)

8. Diop, M., Piponniau, S., Dupont, P.: On the length and time scales of a laminar shock wave boundary layer interaction. American Institute of Aeronautics and Astronautics (2016). DOI 10.2514/6.2016-0073. URL http://dx.doi.org/10.2514/6.2016-0073

9. Durst, F., Melling, A., Whitelaw, J.: Principles and Practice of Laser-Doppler Anemometry (1976)

10. Erdem, E., Kontis, K., Johnstone, E.: Experiments on transitional shock wave-boundary layer interactions at Mach 5 . Experiments in Fluids (2013)

11. Giepman, R.: Flow Control for Oblique Shock Wave Reflections. Ph.D. thesis, Delft University of Technology

12. Giepman, R.H.M., Schrijer, F.F.J., Oudheusden, B.W.: High resolution PIV measurements of a transitional shock wave boundary layer interaction . Experiments in Fluids 56 (2015)
13. Goldstein, R.J., Adrian, R.J.: Measurement of fluid velocity gradients using laser-Doppler techniques. Review of Scientific Instruments 42(9), 1317-1320 (1971)

14. Klebanoff, P.: Characteristics of turbulence in a boundary layer with zero pressure gradient. Tech. rep., NASA (1955)

15. Kreid, D.K.: Laser-Doppler velocimeter measurements in nonuniform flow : error estimates. Appl. Optics 13, 1872 (1974)

16. Larchevêque, L.: Low and Medium Frequency Unsteadinesses in a Transitional Shock-Boundary Reflection with Separation (2016)

17. Le Balleur, J.C., Délery, J.: Etude expérimentale de l'effet de la réflexion d'une onde de choc sur la transition de la couche- limite. In: Congrès Français de Mécanique (1973)

18. Liepmann, H.W.: The interaction between boundary layer and shock waves in transonic flow. Journal of Aeronautical Science 13, 623-637 (1946)

19. Liepmann, H.W., Roshko, A., Dhawan, S.: On reflection of shock waves from boundary layers. NACA Report $1100(1952)$

20. McLaughlin, D.K., Tiederman, W.G.: Biasing correction for individual realization of laser anemometer measurements in turbulent flows. The Physics of Fluids 16(12), 2082-2088 (1973). DOI 10.1063/1.1694269. URL https://aip.scitation.org/doi/abs/10.1063/1.1694269

21. Meinhart, C.D., Wereley, S.T., Santiago, J.G.: A piv algorithm for estimating time-averaged velocity fields. Journal of Fluids Engineering 122(2), 285-289 (2000). DOI 10.1115/1.483256. URL http://dx.doi.org/10.1115/1.483256

22. Melling, A.: The influence of velocity gradient broadening on a mean and rms velocities measured by laser anemometry. Tech. rep.

23. Pate, S., Schueler, C.: Radiated aerodynamic noise effects on boundary-layer transition in supersonic and hypersonic wind tunnels. AIAA Journal 7(8), 450-457 (1969)

24. Piponniau, S., Dussauge, J.P., Debiève, J.F., Dupont, P.: A simple model for low-frequency unsteadiness in shockinduced separation. Journal of Fluid Mechanics 629, 87108 (2009)

25. Raffel, M.: Background-oriented schlieren (BOS) techniques. Experiments in Fluids 56(3), 1-17 (2015). DOI $10.1007 / \mathrm{s} 00348-015-1927-5$. URL http://dx.doi.org/10.1007/s00348-015-1927-5

26. Samimy, M., Lele, S.K.: Motion of particles with inertia in a compressible free shear layer. Physics of Fluids A: Fluid Dynamics 3(8), 19151923 (1991). DOI 10.1063/1.857921. URL https://doi.org/10.1063/1.857921

27. Sansica, A., Sandham, N.D., Hu, Z.: Forced response of a laminar shoc-induced separation bubble. Physics of Fluids (26) (2014)

28. Sansica, A., Sandham, N.D., Hu, Z.: Instability and lowfrequency unsteadiness in a shock-induced laminar separation bubble. J. Fluid Mech. 798, 5-26 (2016)

29. Titchener, N., Colliss, S., Babinsky, H.: On the calculation of boundary-layer parameters from discrete data. Experiments in Fluids 56(8), 159 (2015). DOI $10.1007 / \mathrm{s} 00348-015-2024-5$. URL https://doi.org/10.1007/s00348-015-2024-5 